Methods: A cross-sectional study was performed in 288 LAPR based on online survey about cSLE practices, which included 21 countries. All physicians are members of Pan-American League of Association for Rheumatology (PANLAR). Results: The response rate of web-based survey by LAPR was 170/288 (59\%) and the majority worked in University Hospitals (63\%). The ACR and/or SLICC classification criteria (99\%) and disease activity tools (97\%) were almost universally used by LAPR, whereas damage index $(70 \%)$ and CHAQ $(58 \%)$ instruments were less frequently used. Laboratory exams, diagnostic imaging and biopsies were generally available (>75\%), however low availability for densitometry (66\%) Drug access was excellent for the most common prescribed medications (>75\%), except for belimumab (11\%). Endemic illnesses were reported by LAPR in at least one cSLE patient during the previous year: tuberculosis (16\%) and Hansen disease (2\%). Emerging mosquito-borne diseases were also reported: dengue (20\%), Chikungunya (11\%) and Zika (8\%). Groups were further divided in two, according to the number of cSLE patients followed by LAPR in the last year: group $A$ ( ${ }^{3} 25$ patients) and group $B(<25$ patients). Frequencies of condom in combination with other contraceptive methods were significantly higher in group $\mathrm{A}$ than $\mathrm{B}$ ( $69 \%$ vs. $48 \%, p=0.01)$. The frequencies of reported pregnancy $(50 \%$ vs. $16 \%$ $\mathrm{p}<0.001$ ) and non-adherence to therapy were significantly higher in group $A$ (100\% vs. $93 \%, p=0.023)$. Alcohol intake $(42 \%$ vs. $21 \%, p=0.004)$ and illicit drug use $(19 \%$ vs. $5 \%, p=0.007)$ were also reported more frequently by LAPR of group $A$ in at least one cSLE patient.

Conclusions: This first large web-based survey demonstrated an overall excellent access for diagnosis and therapy by LAPR, probably related to their high rate of practices in tertiary care of University Hospitals. Adherence to therapy, pregnancy and substance abuse were identified as major challenges in this population, particularly in larger centres.

Acknowledgements: This study was supported by grants from Fundação de Amparo à Pesquisa do Estado de São Paulo FAPESP $2015 / 03756-4$ to EB and CAS), Conselho Nacional de Desenvolvimento Científico e Tecnológico (CNPq $305068 / 2014-8$ to EB, 301479/2015-1 to CSM and 303422/2015-7 to CAS).

Disclosure of Interest: J. Ferreira: None declared, V. Trindade: None declared, G. Espada: None declared, Z. Morel: None declared, E. Bonfa: None declared, C. Saad-Magalhães: None declared, C. Silva Grant/research support from: Fundação de Amparo à Pesquisa do Estado de São Paulo FAPESP 2015/03756-4) DOI: 10.1136/annrheumdis-2018-eular.1835

\section{AB1101 THE DEVELOPMENT OF A PALATABLE, STABLE ORAL METHOTREXATE SOLUTION}

\section{J.-A. Penton, J. Winslade, D. Roberts, S. Conroy. Therakind Ltd, London, UK}

Background: Methotrexate is used in the treatment of Rheumatoid Arthritis, Juvenile Idiopathic Arthritis and other conditions such as Acute Lymphoblastic Leukaemia and psoriasis. It can be administered orally or by injection. With oral administration, methotrexate's bitter taste may not be masked, particularly when tablets are crushed to facilitate dosing in paediatrics or patients who have difficulty swallowing tablets. ${ }^{1}$ This can lead to long term treatment adherence issues and inaccurate dosing, putting patients at risk of adverse reactions or inadequate efficacy. A palatable oral liquid presentation of methotrexate would facilitate more accurate dosing and potentially improve treatment adherence; however, methotrexate is difficult to formulate as an oral liquid due to taste, solubility and stability challenges. Despite these difficulties, a palatable, stable oral methotrexate solution has been developed.

Objectives: To develop a palatable, stable, oral methotrexate solution, which complies with EU requirements for development of paediatric products, ${ }^{2}$ to aid accurate dosing and treatment adherence.

Methods: During each of two single-dose (10 mg and $2.5 \mathrm{mg}$ ) bioequivalence studies of the test $2 \mathrm{mg} / \mathrm{ml}$ methotrexate oral solution with licenced tablets, 24 healthy male subjects were asked to comment on the oral solution's taste, indicating whether it was: Bitter, Sour, Salty, Sweet, or No Obvious Taste and they could also leave a remark. In parallel to clinical studies, long term ICH stability studies at ambient conditions $\left(25^{\circ} \mathrm{C} / 60 \% \mathrm{RH}\right)$ and in-use shelf life studies were conducted. Results: All subjects in the $10 \mathrm{mg}$ dose study reported the oral solution as sweet tasting. One subject also reported an aftertaste and strange smell. In the $2.5 \mathrm{mg}$ dose study, all subjects except one reported the solution as sweet tasting. The remaining subject reported a 'soothing' taste. One subject reported it was also sour and another reported an aftertaste. Two subjects additionally commented that they liked the taste. Both studies demonstrated bioequivalence and a similar safety profile between the oral solution and tablets. The stability studies illustrated that the oral methotrexate solution utilised in these clinical studies was stable at ambient conditions for up to 20 months, including a 3 months in-use period. The oral solution has recently been granted a European Union marketing authorisation and is the first methotrexate oral solution to be authorised for treatment of polyarthritic Juvenile Idiopathic Arthritis in Europe.
Conclusions: A $2 \mathrm{mg} / \mathrm{ml}$ oral methotrexate solution, developed to improve treatment adherence and dose accuracy, is reported to have a palatable, sweet taste and can be stored long term at ambient conditions with a 3 months in-use period.

\section{REFERENCES}

[1] 1. Vrignaud S, et al. Int J Pharm 2015;487(1-2):270-3.

[2] EMA/CHMP/QWP/805880/2012 Rev. 2

Acknowledgements: The clinical studies were conducted at PAREXEL. The development work was conducted at Quay Pharmaceuticals.

Disclosure of Interest: J.-A. Penton Employee of: Therakind Ltd, J. Winslade Shareholder of: Therakind Ltd, Employee of: Therakind Ltd, D. Roberts Employee of: Therakind Ltd, S. Conroy Shareholder of: Therakind Ltd, Employee of: Therakind Ltd

DOI: 10.1136/annrheumdis-2018-eular.4596

\section{AB1102 KAWASAKI DISEASE AND GIANT ANEURYSM IN MEXICAN CHILDREN: EVOLUTION AND CLINICAL CHARACTERISTICS: A 5-YEAR EXPERIENCE}

M.T. Brana ${ }^{1}$, S. Osorio ${ }^{2}$, L. Aparicio ${ }^{2}$, A. Rodriguez ${ }^{1}$, E. Faugier ${ }^{2}$, R. Maldonado ${ }^{2}$. ${ }^{1}$ Pediatric Rheumatology, ${ }^{2}$ Hospital Infantil de Mexico, Federico Gomez, Hospital Infantil de Mexico Federico Gomez, Mexico, Mexico

Background: Kawasaki disease (KD) is an acute, self-limited, systemic vasculitis, predominantly involving medium-sized arteries. It mainly affects children younger than five years and it is the leading cause of acquired heart disease in children in developed countries. Of unknown pathogenesis, KD severe complication is the occurrence of coronary artery lesions. Without early treatment, there is a $15 \%$ to $25 \%$ incidence of coronary artery lesions. Management with intravenous immunoglobin (IVIG), combined with aspirin, effectively decrease the incidence of this lesions to a $4 \%$. The long-term prognosis is determined by the initial and current level of coronary artery involvement. Methods to predict which children are at higher risk for coronary aneurysms have been sought to determine prognosis and select patients for more rigorous treatment and follow-up.

Objectives: To describe the clinical presentation and evolution in addition to laboratory findings in Mexican paediatric population who developed giant aneurysms diagnosed with KD during the past 5 years. By identifying mayor risk factors in our population, an effective score could be used to select children for evaluation of additional therapies to prevent coronary artery aneurysms that occur despite treatment with IVIG.

Methods: Retrospective cohort study of the Children's Hospital of Mexico Federico Gomez, last 5 years. We reviewed the data form the clinical archives of the patients who developed giant aneurysms after the diagnosis of KD from 2011 to 2016. A total of 84 patients with KD, 7\% developed giant aneurysms. The variables analysed, apart from the typical clinical and laboratory findings of KD, include size and $\mathrm{Z}$ score of the aneurysms, involution through follow up, cardiac morbidity and mortality, and treatment strategy.

Results: Results: The mean age of patients at diagnostic was 17 months, and $84 \%$ were males. Only $33 \%$ of the patients developed complete KD, while $66 \%$ were diagnosed as incomplete. All patients presented with a positive Harada score. IVIG was administer in $83 \%$ of the patients, and a second dose was needed in $33 \%$. Infliximab was used in $33 \%$ of the patients. One patient died due to cardiogenic shock. Results from echocardiography in the follow-up show that $33 \%$ of the patients have evolved to even larger aneurysms and $50 \%$ present no changes. Of the patients with a longer follow-up, 4 years after diagnostic, 33\% have developed arrhythmias and $16 \%$ myocardial infraction. All are at high risk of sudden death.

Conclusions: The late diagnosis is the characteristic present in all patients which developed giant aneurysms, making imperative to identify clinical and laboratory findings that will help identify KD in Mexican paediatric population to avoid cardiac complications.

\section{REFERENCE:}

[1] Diagnosis, Treatment, and Long-Term Management of Kawasaki Disease: A Scientific Statement for Health Professionals From the American Heart Association. Brian W. McCrindle, et al. Circulation. published online March 29, 2017; Circulation is published by the American Heart Association, 7272 Greenville Avenue, Dallas, TX 75231 Copyright (C) 2017 American Heart Association.

Disclosure of Interest: None declared DOI: 10.1136/annrheumdis-2018-eular.1879 